\title{
Outbreak of bloodstream infection with extended- spectrum ß-lactamase-producing Klebsiella pneumoniae at a Teaching Hospital
}

\author{
Senanayake NP1 , Karunanayak $L^{2}$
}

\begin{abstract}
Introduction: Klebsiella pneumoniae (K. pneumoniae) is an important hospital-acquired pathogen causing severe infections in neonatal units. Several outbreaks of infection caused by multi-drug-resistant $K$. pneumoniae isolates have been widely reported among neonates. The aim is to investigate an outbreak of blood stream infection caused by Extended-Spectrum ß-Lactamases (ESBL) producing K.pneumoniae in a Neonatal Intensive Care Unit (NICU) at Teaching Hospital Kandy, Sri Lanka. Materials and Methods: Blood culture samples were collected from the neonates on admission to the NICU and 2 to 3 days later on clinical suspicion of blood stream infection. The blood culture samples were processed according to the standard methods and the antibiotic susceptibility tests were carried out as per Clinical Laboratory Standards Institute (CLSI) guidelines. Results: Of the 140 blood cultures 36 were identified as Extended-Spectrum ß-Lactamases (ESBL) producing K.pneumoniae. All the isolates were susceptible to ciprofloxacin, amikacin, netilmicin, imipenem and meropenem. Twenty eight of the 36 patients responded to treatment with a combination of amikacin and meropenem. Conclusion: ESBL producing K.pneumoniae was responsible for this outbreak at the NICU. Knowing the susceptibility patterns of clinical isolates will allow the rational use of antibiotics, which is important in the treatment of infections with multi-drugresistant bacteria.
\end{abstract}

Key words: Blood stream infections, Klebsiella pneumoniae, Neonatal intensive care unit

\section{Introduction}

K ebsiella pneumoniae is an important hospital-acquired pathogen with the potential of causing severe infections in neonatal units $^{1,2}$. For neonates in high-dependency units, hospital acquired bloodstream infections are an important cause of morbidity and mortality ${ }^{3}$.

Several outbreaks of infection caused by $K$. pneumoniae isolates that are simultaneously resistant to broad-spectrum cephalosporins and aminoglycosides have been widely reported ${ }^{1}$.

Some of these multidrug-resistant isolates produce extendedspectrum ß-lactamases (ESBLs) that are able to hydrolyze extended-
'Dr. Nilanthi Priyadharshika Senanayake, MBBS, Diploma in Mediical Microbiology, MD in Medical Microbiology Registrar in Microbiology, Department of Micriobiology, Teaching Hospital kandy, ${ }^{2}$ Dr. Lilani Karunanayak, MBBS, Diploma in Medical Microbiology, MD in Medical Microbiology.

\author{
Address for correspondence: \\ Department of Para Clinical sciences, \\ Faculty of Medicine, General Sir John Kotelawala \\ Defence University, Sri Lanka \\ Personal address: No 14, Bodhiraja Mawatha, \\ Jayanthipura, Battaramulla, Sri Lanka
}

\section{How to cite}

Senanayake NP, Karunanayak L. Outbreak of bloodstream infection with extended-spectrum B-lactamase-producing Klebsiella pneumoniae at a Teaching Hospital. J Nepal Paediatr Soc 2014;34(3):230-232.

doi: http://dx.doi.org/10.3126/jnps.v34i3.10459

This work is licensed under a Creative Commons Attribution 3.0 License.

spectrum cephalosporins (eg. ceftriaxone, cefotaxime, and ceftazidime), aztreonam, and related oxyimino-B-lactams. (4)

The objective of this study was to investigate an outbreak of blood stream infection caused by extendedspectrum ß-lactamases (ESBL) producing K.pneumoniae in a neonatal intensive care unit (NICU) at Teaching Hospital Kandy, Sri Lanka during the period December 2009 to January 2010. 


\section{Materials and Methods}

This study was carried out at the NICU at the Teaching Hospital Kandy, Sri Lanka, which experienced an outbreak of blood stream infections during the period $2^{\text {nd }}$ December 2009 to $17^{\text {th }}$ January 2010.

Blood culture samples were taken from all the neonates who were admitted to the NICU during this period. The blood cultures were taken on admission to the NICU and 2 to 3 days later on clinical suspicion of blood stream infection. The clinical diagnosis or suspicion of neonatal septicaemia was made by the paediatric unit responsible for the care of the patient.

Blood culture samples were collected aseptically and bottles showing evidence of growth after overnight incubation at $37^{\circ} \mathrm{C}$ were promptly sub-cultured into MacConkey, Blood and chocolate agar media and incubated in appropriate temperature, atmospheres according to established methods. The clinical isolates were identified by Gram's staining, colony characteristics and the findings were confirmed by the API 20 E system. The antibiotic susceptibility tests and the detection of ESBL were carried out as per Clinical Laboratory Standards Institute (CLSI) guidelines.

\section{Results}

During this period 145 neonates were admitted to the NICU at Teaching Hospital Kandy, and a total of 140 blood cultures were collected from them. All the initial blood culture samples collected from the neonates on admission to the NICU were negative. Seventy six (54.28\%) of the 140 blood culture samples which were collected 2 to 3 days after admission to the NICU became positive for bacterial growths, while 64 (45.71\%) yielded no growth. Of the 76 positive cultures $48(63.15 \%)$ were pure growths. Of the 48 pure cultures $36(75 \%)$ isolates were identified as ESBL producing K.pneumoniae and $12(25 \%)$ isolates were identified as coagulase negative staphylococcal species.

All the isolates of $K$ pneumoniae shared the in vitro susceptibility to gentamicin, amikacin, netilmicin, imipenem and meropenem. All the clinical isolates were resistant to cefotaxime, ceftazidime and ceftriaxone.

Twenty eight of the 36 patients responded to treatment with a combination of amikacin and meropenem. Eight of the 36 patients died of sepsis in spite of appropriate antibiotics.

\section{Discussion}

The Neonatal Intensive Care Unit (NICU) at the Teaching Hospital Kandy, Sri Lanka experienced an outbreak of 36 cases of K.pneumoniae blood stream infections during the period $2^{\text {nd }}$ December 2009 to $17^{\text {th }}$ January 2010.

The most significant finding of this study was that all the Klebsiella pneumoniae isolates were resistant to commonly used antibiotics and were extended spectrum ß-lactamase producers (ESBL).

The significant morbidity and mortality arising from intra-hospital infection with multi-drug-resistant strains of K.pneumoniae, as seen in this report, is reason for alarm.

The high incidence of ESBL producing K.pneumoniae septicaemia and the antibiotic susceptibility pattern, indicate that the infection was most probably nosocomial in origin.

It appears that the lack of control of contamination sources and hand hygiene has caused the dissemination of the microorganisms among patients. However, there was no direct evidence of the source of contamination or transmission of the multidrug-resistant strains, although it was possibly due to transitory hand contact through health care workers ${ }^{5}$.

\section{Conclusion}

Knowing the susceptibility and resistance patterns in these types of clinical isolates will allow the implementation of improved therapeutic measures and the rational use of antibiotics, which are indispensable tools in fighting infectious diseases caused by multidrug resistant bacteria.

Acknowledgements: Nil

Funding: Nil

Conflict of Interest: None

Permission from IRB: Yes

\section{References}

1. French GL, Shannon KP, Simmons P. Hospital outbreak of Klebsiella pneumoniae resistant to broad-spectrum cephalosporins and B-lactamblactamase inhibitor combinations by hyper production of SHV-5-B-lactamase. J Clin Microbiol 1996;34:358-363. 
2. Rice LB, Eckstein EC, DeVente J, Shlaes DM. Ceftazidime-resistant Klebsiella pneumoniae isolates recovered at the Cleveland Department of Veterans Affairs Medical Center. Clin Infect Dis 1996;23:118-124.

3. Podschun R, Ullmann U. Klebsiella spp. As nosocomial pathogens: epidemiology, taxonomy, typing methods, and pathogenicity factors. Clin Microbiol Rev 1998;11:589-603.
4. Medeiros AA. Evolution and dissemination of B-lactamases accelerated by generations of B-lactam antibiotics. Clin Infec Dis 1997;24 (Suppl. 1):S19-S45.

5. Brun-Buisson C, Legrand P, Philippon A, Montravers F, Ansquer M, Duval J. Transferable enzymatic resistance to third-generation cephalosporins during nosocomial outbreak of multiresistant Klebsiella pneumoniae. Lancet 1987;2(8554):30206. 